\title{
Tantalates Transcend Titanates
}

\section{Two-dimensional superconductivity and anisotropic transport at $\mathrm{KTaO}_{3}$ (111) interfaces}

Authors: Changjiang Liu, Xi Yan, Dafei Jin, Yang Ma, Haw-Wen Hsiao, Yulin Lin, Terence M. Bretz-Sullivan, Xianjing Zhou, John Pearson, Brandon Fisher, J. Samuel Jiang, Wei Han, Jian-Min Zuo, Jianguo Wen, Dillon D. Fong, Jirong Sun, Hua Zhou, Anand Bhattacharya

Science 371, 716 (2021)

2. Two-Dimensional Superconductivity at the $\mathrm{LaAlO}_{3} / \mathrm{KTaO}_{3}[110]$ Heterointerface

Authors: Zheng Chen, Zhongran Liu, Yanqiu Sun, Xiaoxin Chen, Yuan Liu, Hui Zhang, Hekang Li, Meng Zhang, Siyuan Hong, Tianshuang Ren, Chao Zhang, He Tian, Yi Zhou, Jirong Sun, and Yanwu Xie

Phys. Rev. Lett. 126, 026802 (2021)

\section{Superconductor-Metal Quantum Transition at the EuO/ $\mathrm{KTaO}_{3}$ Interface} Authors: Yang Ma, Jiasen Niu, Wenyu Xing, Yunyan Yao, Ranran Cai, Jirong Sun, X. C. Xie, Xi Lin, and Wei Han

Chin. Phys. Lett. 37, 117401 (2020)

\section{Recommended with a Commentary by Dirk van der Marel, University of Geneva}

$\mathrm{KTaO}_{3}$ and $\mathrm{SrTiO}_{3}$ are band insulators with a $3 \mathrm{eV}$ gap. At room temperature they have the cubic perovskite structure and both materials are close to a ferroelectric instability [1]. The first available bands above the gap are the three-fold degenerate $t_{2 g}$ states $\left(d_{x y}, d_{y z}\right.$ and $d_{z x}$ ) derived from the $\mathrm{Ti} 3 d$ shell in the case of $\mathrm{SrTiO}_{3}[2]$ and from the Ta $5 d$ shell in the case of $\mathrm{KTaO}_{3}[4,5]$. At $k=0$ spin-orbit coupling lifts one of the three bands above the other two, namely $20 \mathrm{meV}$ for $\mathrm{SrTiO}_{3}$ and $0.4 \mathrm{eV}$ for $\mathrm{KTaO}_{3}$. The two lowest bands have different energy-momentum dispersion, giving rise to one "heavy" and one "light" band that coincide at $k=0$. For $\mathrm{SrTiO}_{3}$ a tetragonal distortion below $105 \mathrm{~K}$ causes a tiny splitting at $k=0$ between the lowest two bands [2,3] and superconductivity is observed when one, two or all three $t_{2 g}$ bands are partly occupied, with $T_{c} \leq 0.4 \mathrm{~K}$ for the bulk material [6], and $\leq 0.3 \mathrm{~K}$ for 2 dimensional $\mathrm{SrTiO}_{3} / \mathrm{LaAlO}_{3}$ interfaces [7]. For $\mathrm{KTaO}_{3}$ the structure remains simple cubic down to $0 \mathrm{~K}$, and for all relevant dopings two bands are occupied while the third band remains empty. In 2011 Ueno et al. [8] reported that electron doped $\mathrm{KTaO}_{3}$ becomes superconducting below $50 \mathrm{mK}$. 
Enter three remarkable papers, submitted in december 2019 (Liu et al.), april 2020 (Chen et al.), september 2020 (Ma et al.) and published in reverse order [9].

1. The first system studied by Liu et al. was amorphous $\mathrm{LaAlO}_{3}$ deposited on the (111) surface of $\mathrm{KTaO}_{3}$. They observed the superconducting transition for different dopings at $T_{c}\left(7 \times 10^{13}\right)=1.47 \mathrm{~K}$, and at $T_{c}\left(8.9 \times 10^{13}\right)=1.14 \mathrm{~K}$. Here the number of electrons per $\mathrm{cm}^{2}$ is indicated in the brackets.

2. The second system studied by Liu et al. was polycrystalline EuO deposited on the (111) surface of $\mathrm{KTaO}_{3}$, for which they observed the superconducting transition for different dopings at $T_{c}\left(1.04 \times 10^{14}\right)=2.2 \mathrm{~K}, T_{c}\left(9.9 \times 10^{13}\right)=1.86 \mathrm{~K}, T_{c}\left(9.2 \times 10^{13}\right)=1.74 \mathrm{~K}$, and $T_{c}\left(6 \times 10^{13}\right)=1.26 \mathrm{~K}$. Ma et al. also studied this system and found the superconducting transition at $T_{c}\left(7.4 \times 10^{13}\right)=1.33 \mathrm{~K}$, which fits nicely in the doping dependence reported by Liu et al..

3. Chen et al. studied amorphous $\mathrm{LaAlO}_{3}$ deposited on the (110) surface of $\mathrm{KTaO}_{3}$ and observed the superconducting transition at $T_{c}\left(7 \times 10^{13}\right)=0.9 \mathrm{~K}$.

4. The third system studied by Liu et al. was amorphous $\mathrm{LaAlO}_{3}$ deposited on the (001) surface of $\mathrm{KTaO}_{3}$, which did not result in superconductivity down to $0.025 \mathrm{~K}$.

Additional intriguing observations concern a transport anisotropy above $T_{c}$, suggesting the emergence of a distinct "stripe"-like phase, which is also revealed near the critical field. Ma et al. discuss this behavior in the context of a quantum Griffiths singularity due to quenched disorder at the interface, which they attribute to the polycrystalline properties of the EuO layer. More experiments are needed to sort out this effect and the conditions under which it occurs.

The elephant in the room is of course the factor 5 higher $T_{c}$ of doped $\mathrm{KTaO}_{3}$ compared to $\mathrm{SrTiO}_{3}$. Everything indicates that the only relevant interactions are the Coulomb interaction and coupling of the electrons to the lattice degrees of freedom. In other words, at first glance these materials appear to be garden variety superconductors where the pairing is mediated by electron-phonon coupling. And yet. Doped $\mathrm{SrTiO}_{3}$ was originally predicted to be a multivalley superconductor. The doped material was found to be superconducting all-right, but it has also become overwhelmingly clear that the bandstructure is not of the multi-valley variety.

A recent theory predicted a giant isotope effect of $T_{c}$ due to a pairing interaction mediated by the ferro-electric soft modes [10]. The giant isotope effect has been observed all-right [11, $12,13]$, and a similar effect was observed by using $C a$ substitution to tune the ferro-electric instability [14]. However, it has also become overwhelmingly clear that for the low carrier concentrations in these superconductors the electron-phonon coupling to the ferro-electric soft modes becomes vanishingly small as a result of parity selection rules [15]. These selection rules do not exclude two-phonon exchange processes. Such processes have been identified as particularly prominent in $\mathrm{SrTiO}_{3}[16,17]$, and applying the same arguments as in Ref. [17] they should be stronger still in $\mathrm{KTaO}_{3}$.

The $T_{c}(n)$ phase diagram of $\mathrm{SrTiO}_{3}$ has been fully reproduced assuming coupling to plasmons and LO1 modes $[18,19,20,21]$. The weak point of this approach is, that at least 
one of the two ingredients (coupling to plasmons) relies on an incomplete treatment of the electron gas: A purely electronic mechanism, i.e. pairing in the electron gas mediated by the screened Coulomb interaction (which includes coupling to plasmons), has been studied over the past decades in increasing detail $[22,23,24,25,26,27,28,29]$. While the initial results of Takada looked promising and superconducting was predicted for $r_{s}>6$ (which for $\mathrm{SrTiO}_{3}$ corresponds to $n<4 \cdot 10^{17}$ ), accounting for exchange and correlation energy showed that no superconductivity is present for $r_{s}<10$ [29], and $r_{s}<30$ [27]. Since the transition to the ferromagnetic phase occurs already at about $r_{s}=25[30,31,32]$ and Wigner crystallization at $r_{s}=106[30,32]$ the superconducting state appears not to be stable in the electron gas.

Since there is overwhelming reason to believe that the same physics is at work in $\mathrm{KTaO}_{3}$ and in $\mathrm{SrTiO}_{3}$, we can look at commonalities and differences between the two materials to decide empirically which aspects are important and which ones aren't. Among other things the number of partly occupied bands doesn't appear to matter. The effective mass of the "heavy" conduction band (and therefor the density of states) is smaller for $\mathrm{KTaO}_{3}$ than for $\mathrm{SrTiO}_{3}$. Apparently whatever mediates the superconductivity does not seem to profit from a higher density of states.

The observations of superconductivity up to $2.2 \mathrm{~K}$ in electron-doped $\mathrm{KTaO}_{3}$ comes as a surprise. The low charge carrier density poses a theoretical challenge. Nevertheless, this looks like a nut that someone should be able to crack.

\section{References}

[1] H. Fujishita et al., J. Phys. Soc. Japan 85, 074703 (2016).

[2] D. van der Marel et al., Phys. Rev. B 84, 205111 (2011).

[3] X. Lin, Phys. Rev. Lett. 112, 207002 (2014).

[4] P. D. C. King et al., Phys. Rev. Lett. 108, 117602 (2012).

[5] F. Y. Bruno et al., Advanced Electronic Materials 5, 1800860 (2019).

[6] C. S. Koonce et al., Phys. Rev. 163, 380 (1967).

[7] N. Reyren et al., Science 317, 1196 (2007).

[8] K. Ueno et al., Nature Nanotechnology 6, 408 (2011).

[9] N. N., $\epsilon v \alpha \gamma \gamma \epsilon \lambda \iota \circ \nu \kappa \alpha \tau \alpha \mu \alpha \theta \prime \theta \alpha \iota 0 \nu$ 20:16 ( 85).

[10] J. M. Edge et al., Phys. Rev. Lett. 115, 247002 (2015).

[11] A. Stucky et al., Scientific Reports 6, 37582 (2016).

[12] Y. Tomioka et al., Nature Comm. 10, 738 (2019).

[13] W. Rischau et al., submitted (2021). 
[14] C. W. Rischau et al., Nature Physics 13, 643 (2017).

[15] J. Ruhman and P. A. Lee Phys. Rev. B 100, 226501 (2018).

[16] K. L. Ngai, Phys. Rev. Lett. 32, 215 (1974).

[17] D. van der Marel et al., Phys. Rev. Res. 1, 013003 (2019).

[18] Y. Takada, J. Phys. Soc. Japan 49, 1267 (1980).

[19] J. Ruhman and P. A. Lee, Phys. Rev. B 94, 224515 (2016).

[20] S. N. Klimin et al., J. Superconductivity and Novel Magnetism 32, 2739 (2019).

[21] C. Enderlein et al., Nature Comm. 11, 4852 (2020).

[22] Yasutami Takada, J. Phys. Soc. Japan 45, 786 (1978).

[23] B. Schuh and L. J. Sham, J. Low Temp. Phys. 50, 391 (1983).

[24] H. Rietschel and L. J. Sham, Phys. Rev. B 28, 5100 (1983).

[25] T. Buche and H. Rietschel, Phys. Rev. B 41, 8691 (1991).

[26] M. Grabowski and L. J. Sham, Phys. Rev. B 29, 6132 (1984).

[27] S. Kuechenhoff and P. Woelfle, Phys. Rev. B 38, 935 (1988).

[28] S. Kurth et al., Phys. Rev. Lett. 83, 2628 (1999).

[29] M. Wierzbowska and J. W. Krogh, Phys. Rev. B 71, 014509 (2005).

[30] D. M. Ceperley and B. J. Alder, Phys. Rev. Lett. 45, 566 (1980).

[31] G. Ortiz, M. Harris, and P. Ballone, Phys. Rev. Lett. 82, 5317 (1999).

[32] N. D. Drummond and R. J. Needs, Phys. Rev. Lett. 102, 126402 (2009). 